\title{
Erratum
}

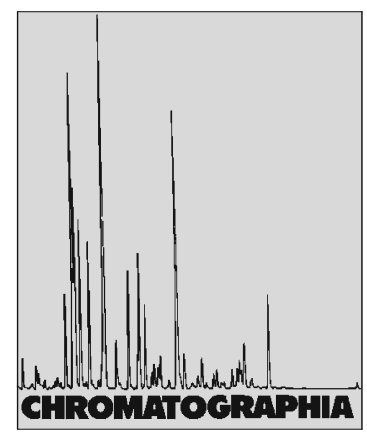

$2009,69,601$

\section{GC Analysis of the Fatty Acid Composition of Yak Kidney}

Qiang Wang ${ }^{1}$, Jianping $\mathrm{Wu}^{2,3, 凶}$, Shenggui Zhang ${ }^{1}$, Yubin Zhang ${ }^{1}$, Haixia Zhang ${ }^{4}$, Enguo Fan ${ }^{1,5, 凶}$

${ }^{1}$ College of Food Science and Engineering, Gansu Agricultural University, 730070 Lanzhou, China

${ }^{2}$ College of Animal Science and Technology, Gansu Agricultural University, 730070 Lanzhou, China

${ }^{3}$ Center for Engineering and Technological Research on Livestock Products of Gansu Province, 730070 Lanzhou, China; E-Mail: jp-wu@hotmail.com

${ }^{4}$ College of Agronomy, Gansu Agricultural University, 730070 Lanzhou, China

5 Institut für Biochemie und Molekularbiologie, Universität Freiburg, Stefan-Meier-Strasse 17, 79104 Freiburg, Germany;

E-Mail: enguo.fan@biochemie.uni-freiburg.de

\section{Erratum to: Chromatographia (2009) 69:139-143 DOI 10.1365/s10337-008-0845-6}

Unfortunately, in the original article in the "Experimental"/"GC Analysis" a wrong column is specified.

FAME analysis was performed with a Clarus500 gas chromatograph (Perkin-Elmer, USA) equipped with a CP Sil 88 column $(120 \mathrm{~m} \times 0.32 \mathrm{~mm}$ i.d.; film thickness $0.2 \mu \mathrm{m})$.

The actual column is a SGE-FFAP column $(120 \mathbf{~ m} \times \mathbf{0 . 2 5} \mathbf{~ m m}$ i.d.; film thickness $\mathbf{0 . 2 5} \boldsymbol{\mu m})$.

The online version of the original article can be found under doi:10.1365/s10337-0080845-6. 